\title{
3D Needle-Tissue Interaction Simulation for Prostate Brachytherapy
}

\author{
Orcun Goksel ${ }^{1}$, Septimiu E. Salcudean ${ }^{1}$, Simon P. DiMaio ${ }^{2}$, \\ Robert Rohling ${ }^{1}$, and James Morris ${ }^{3}$ \\ 1 Department of Electrical and Computer Engineering, \\ University of British Columbia, Vancouver, Canada \\ \{orcung, tims, rohling\}@ece.ubc.ca \\ 2 Surgical Planning Laboratory, Department of Radiology, \\ Brigham and Women's Hospital, Boston, USA \\ 3 Vancouver Center, British Columbia Cancer Agency, Vancouver, Canada
}

\begin{abstract}
This paper presents a needle-tissue interaction model that is a $3 \mathrm{D}$ extension of a prior work based on the finite element method. The model is also adapted to accommodate arbitrary meshes so that the anatomy can effectively be meshed using third-party algorithms. Using this model a prostate brachytherapy simulator is designed to help medical residents acquire needle steering skills. This simulation uses a prostate mesh generated from clinical data segmented as contours on parallel slices. Node repositioning and addition, which are methods for achieving needle-tissue coupling, are discussed. In order to achieve realtime haptic rates, computational approaches to these methods are compared. Specifically, the benefit of using the Woodbury formula (matrix inversion lemma) is studied. Our simulation of needle insertion into a prostate is shown to run faster than $1 \mathrm{kHz}$.
\end{abstract}

\section{Introduction}

Prostate cancer is the most common cancer in the US with 232,090 new cases and 30,350 deaths estimated in 2005. Brachytherapy is the single most commonly used curative treatment for prostate cancer in North America. It is a conformal radiation therapy method that involves the percutaneous insertion of radioactive sources directly in or adjacent to malignant tissues. A conceptual overview of the procedure can be seen in Fig.11.

Despite the low risk of brachytherapy, seed placement errors are still common. In [1], an experienced physician implanting seeds in 20 patients achieved an average placement error of $0.63 \mathrm{~cm}$, which is a substantial error of $21 \%$ of average prostate diameter $(3 \mathrm{~cm})$. Errors decrease the effectiveness of the treatment and may also lead to subsequent complications such as urinary incontinence and impotence. Some procedural challenges of brachytherapy are needle steering and visualizing the $3 \mathrm{D}$ volume from $2 \mathrm{D}$ medical images. The required skill set is acquired by medical residents using mannequins, animals, or cadavers, which are either not very realistic or may involve ethical issues. Residents also practice 

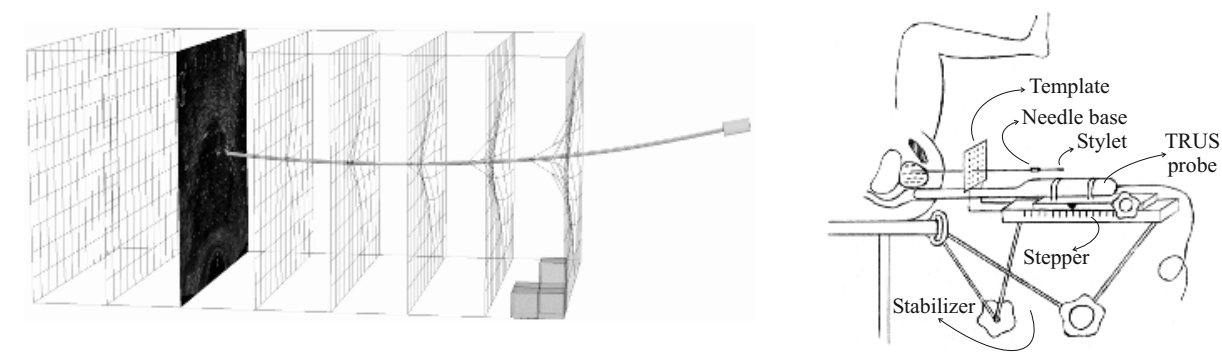

Fig. 1. Representation of 3D prostate volume deformation with a needle inserted (left) and conceptual overview of the procedure (right)

on patients which may lead to possibly irreversible consequences. The identification of this need for better training schemes led us to propose a prostate brachytherapy simulator.

The simulation of needle insertion differs from the simulation of other medical tool-tissue interactions in several ways: the needle does not manipulate only the organ surface; friction is a significant force during its interaction; and it is flexible unlike many rigid surgical tools. These issues were addressed in the 2D case with a finite element model (FEM) using the condensation approach, a stick-slip friction model, and local frame rotations with low-rank matrix updates, respectively [2]. Fluid pockets were then introduced onto this system [3].

Online mesh modification is a common challenge of real-time implementations (e.g., incision in [4]). To retain tissue mesh nodes on the needle, element subdivision and node relocation techniques were implemented in [2, 4, 5]. Specifically, the 2D haptic implementation in [2] simply constrains the closest tissue node onto the needle without any processing of the nominal mesh which locally deforms the tissue unrealistically. This work was also the first to model a decoupled tip cutting force and a velocity-dependent shaft friction force.

There are models [6] and graphical interfaces [7] designed for prostate brachytherapy. However, the only available physically-based brachytherapy model [5] is designed for offline planning simulation and it runs in 2D neglecting spatial deformations such as transversal rotation. In contrast, our proposed model runs at haptic rates while simulating needle insertion in 3D. Extension of algorithms from $2 \mathrm{D}$ to $3 \mathrm{D}$ is not a trivial task. It also increases the computational complexity significantly. Control of such a 3D simulation at haptic rates require additional computational treatment on the algorithms, some of which are presented in this paper. An application on a clinical case such as brachytherapy needs to also address issues related to optimal discretization of the tissue for the model.

\section{Methods}

In the simulation, while the needle is inserted, it interacts with the tissue through tissue mesh nodes that are confined onto the needle shaft. These nodes are called 
contact nodes. When the needle base is manipulated by the user, the needle shaft poses a displacement boundary condition for the tissue mesh via contact nodes. The reaction forces of the tissue are then applied back on the needle model to solve for needle bending and to find feedback forces onto the user's hand. The following steps were taken to implement this system in 3D.

\subsection{Mesh Generation}

Considering FEM is an approximation technique for elastic deformation, its approximation error largely depends on the element discretization geometry. This can be achieved simply by using more elements. However, the speed of many mesh-processing algorithms is significantly affected by the number of nodes. These pose the two basic constraints: a high quality mesh with few nodes. A case with multiple sub-regions with different physical properties, in addition, necessitates a conforming mesh having element surfaces as boundaries between separate anatomical structures. Considering that the segmentation by the physician is simply in the form of parallel contours, generating a prostate region mesh obeying the above criteria requires additional steps, since the conforming meshing algorithms work on a boundary surface definition. Thus, the following steps are taken on the segmentation of the parallel images from a prostate volume study: first the extraneous nodes are removed, then Nuages [8] is used to obtain the surface definition, and finally the interior (with finer elements) and the exterior (with coarser elements) of the prostate are meshed (see Fig.21) using the academic version of GiD [9] which utilizes the advancing fronts technique.

The needle bending is modeled using FEM with quadratic strain. Its deflection is solved using the iterative approach of [2]. The deflection response of this model to an applied force was observed to be substantially mesh topology dependent in 3D. Therefore, the symmetric needle segment in Fig.2 was designed. A needle model constructed from segments similar to the one shown demonstrates axial symmetry for a change of lateral force direction. On the tissue interaction side, this mesh model is assumed to be a set of line segments passing through its center. The needle-tissue coupling is damped in order to ensure stability.
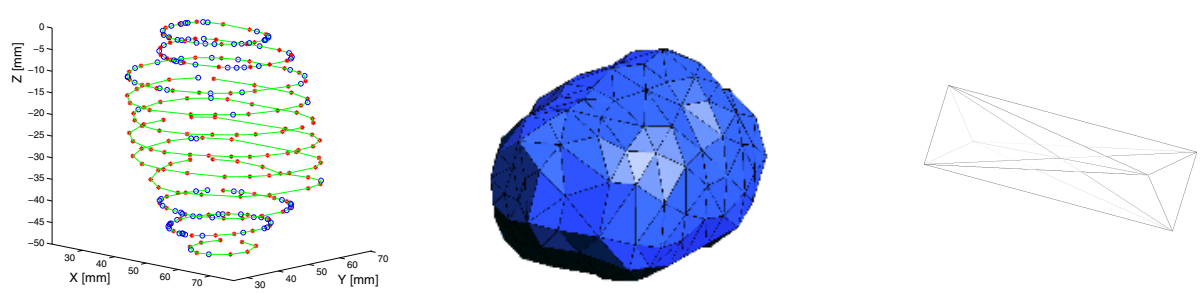

Fig. 2. Prostate segmentation data where the lines denote contours, stars are culled nodes and the circles are the removed ones (left); prostate surface definition (center); and our symmetric design of a needle segment (right) 


\subsection{Implementation}

As opposed to the surrounding relatively soft tissue, a stiffer region connects the prostate to the pubic arch from its anterior inferior end. This creates a torquing movement of the prostate as the needles are inserted. To simulate this effect, the prostate is spatially fixed around this region by setting fundamental displacement constraints. Then the tissue stiffness matrix $\boldsymbol{K}$ is inverted offline before the simulation starts. A linear quasi-static tissue model is used as an approximation to the deformation characteristics of soft tissue. An overview of the system is given in Fig. 3.

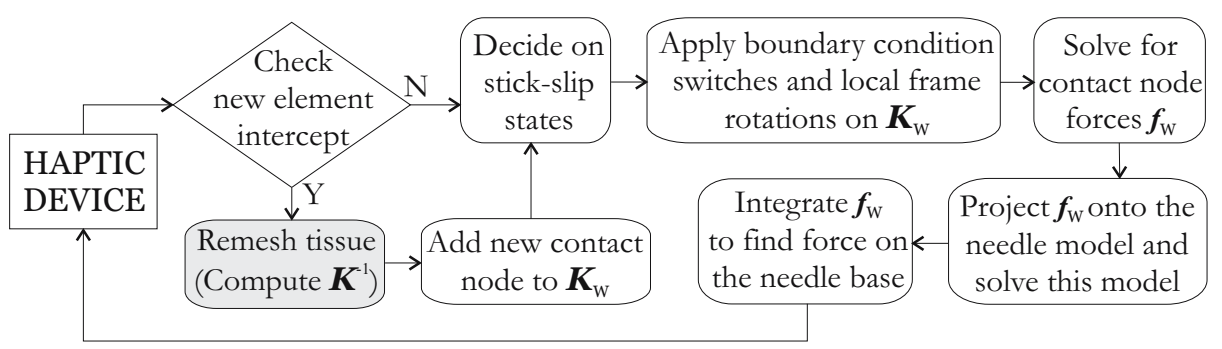

Fig. 3. Flowchart of an iteration during the needle insertion into the tissue

Considering that the needle boundary conditions are applied only on the contact nodes and the feedback forces for the needle are only required on those nodes, a $3 w \times 3 w$ condensed system can be employed with the equation:

$$
\boldsymbol{x}=\boldsymbol{K}_{\mathrm{W}} \boldsymbol{y}
$$

where $\boldsymbol{K}_{\mathrm{W}}$ is the condensed stiffness matrix and $w$ is the number of contact nodes. This condensation approach and the stick-slip friction model applied on it are extended to 3D from the work of [2]. A switch in the stick-slip state of a node is achieved by changing its axial boundary constraint. A force-displacement boundary constraint switch (BCS) on row/column $i$ is in the form of the following low-rank update:

$$
\boldsymbol{K}_{\mathrm{W}}{ }^{\prime}=\boldsymbol{K}_{\mathrm{W}}-\frac{1}{k(i, i)} \boldsymbol{c}_{i} \boldsymbol{r}_{i}
$$

where $\boldsymbol{c}_{i}$ and $\boldsymbol{r}_{i}$ are the corresponding $i^{\text {th }}$ column and $i^{\text {th }}$ row of $\boldsymbol{K}_{\mathrm{W}}$, and $k(i, i)$ is the pivot $i$. Note that this requires $\mathcal{O}\left(w^{2}\right)$ operations. With the changing orientations of the needle segments caused by either user manipulation or needle shaft bending, $\boldsymbol{K}_{\mathrm{W}}$ needs to be updated to accommodate the new local frame orientations. This local frame rotation of a contact node differs depending on its stick-slip state. A treatment in 3D is developed based on the 2D case in [2].

The tissue reaction forces of the contact nodes are linearly distributed onto the closest needle mesh nodes to be applied onto the needle model and also to determine force/torque feedback to the user's hand. 


\section{$2.3 \quad$ Tissue Remeshing}

Although it is possible to maintain a non-conforming needle-tissue coupling using force/displacement interpolations, in our insertion simulation, preserving this conformity was preferred due to speed considerations. It is achieved by placing a tissue node on the needle tip whenever it encounters a new tissue element. Generation of this node without distorting the tissue requires remeshing (the shaded step in Fig. 31). Two remeshing techniques, node repositioning and node addition, are shown in Fig.4. Repositioning is equivalent to removing the surrounding elements of $P$ and introducing the elements surrounding the new node $P^{\prime}$. In contrast, addition in 2D is removing only two elements and introducing four smaller ones (see Fig.4(d)) .

The 3D simulation uses the inverse of $3 n \times 3 n$ tissue stiffness matrix $\boldsymbol{K}$ where $n$ is the number of tissue mesh nodes. Considering the dimensions of $\boldsymbol{K}^{-1}$, a realtime full-rank inversion is impractical. Thus, two methods of fast $\boldsymbol{K}^{-1}$ manipulation are implemented and compared. The first one employs boundary condition switches whereas the second one is a one-step algebraic solution. Nonetheless, both approaches require the differential $3 m \times 3 m$ stiffness matrix $\Delta K$ which is the desired change in $\boldsymbol{K}$ to obtain the remeshed stiffness matrix $\boldsymbol{K}^{\prime}$ where $m$ is the total number of nodes involved. $\Delta \mathrm{K}$ can easily be found by subtracting the stiffness matrices of the elements to be removed from the ones to be added. Note that for the $2 \mathrm{D}$ cases in figures $4(\mathrm{c})$ and (d), $m$ is 7 and 5 , respectively.

The first method performs boundary condition switches on $\boldsymbol{K}^{-1}$ for all three axes of each node involved using (2). It then adds $\Delta \mathrm{K}$ and switches the boundary conditions back. This results in $\approx 2(3 m)(3 n)^{2}$ operations. Throughout this paper, operations refer to floating-point multiplications and divisions only. On the other hand, the second method employs the Woodbury formula as follows:

$$
\left(\boldsymbol{K}+V \Delta \mathrm{K} V^{T}\right)^{-1}=\boldsymbol{K}^{-1}-\left[\boldsymbol{K}^{-1} V \Delta \mathrm{K}\left(I+V^{T} \boldsymbol{K}^{-1} V \Delta \mathrm{K}\right)^{-1} V^{T} \boldsymbol{K}^{-1}\right]
$$

where $V$ is a $3 n \times 3 m$ zero matrix with $3 m$ corresponding rows taken from identity matrix $I_{3 m \times 3 m}$. A fast implementation of (3) with the low-rank inverse computed using Gaussian elimination costs $\approx 3 m(3 n)^{2}+(3 m)^{2} 3 n+2(3 m)^{3}$ operations.

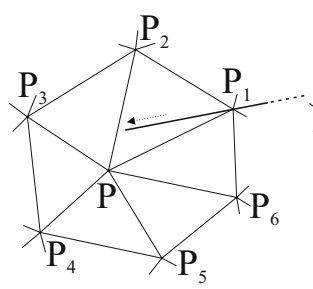

(a)

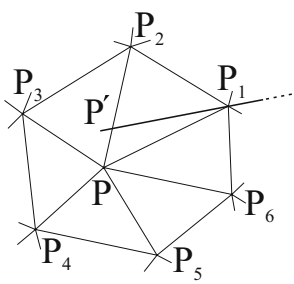

(b)

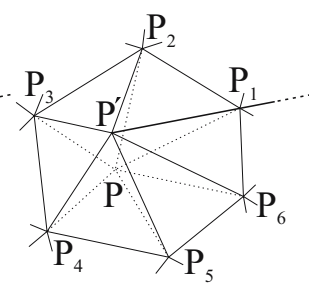

(c)

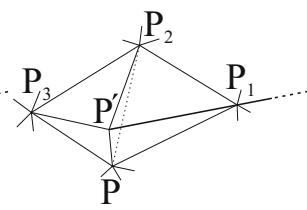

(d)

Fig. 4. The simulation steps (a) before and (b) after a new element penetration; and (c)node repositioning and (d) node addition methods for preserving mesh conformity 
Although $n$ is the dominant factor, the speed of both approaches (BCS and Woodbury) is also dependent on the dimension of $\Delta \mathrm{K}$. For node repositioning, this dimension is determined by node valence which causes a relatively long and unpredictable computation time. Furthermore, repositioning an organ boundary node may pose the issue of distorting the anatomy. In contrast, 3D node addition runs at constant time with a guaranteed $\Delta \mathrm{K}$ size of $18 \times 18$. The extra rows/columns needed in $\boldsymbol{K}^{-1}$ by the Woodbury formula for the addition of $P^{\prime}$ are initially set to zero except for their diagonal elements having very large values to represent infinity. The condition number of the low-rank matrix inverted in (3) is also considered while determining these large numbers. Everytime the needle is completely out of the tissue, $\boldsymbol{K}^{-1}$ is reset to its initial dimensions and values in order not to crowd the model with nodes after consecutive insertions.

\section{Results and Discussion}

The two computational approaches, BCS and Woodbury, were implemented on a 2D tissue (see Fig. 5) in Matlab ${ }^{\circledR}$ using a P4 3.2 GHz system with 1 MB L2 cache. Although both techniques theoretically have the same order of complexity (for $n \gg m$ ), it is observed that the Woodbury formula update runs significantly faster for large $\boldsymbol{K}^{-1}$ (see Fig. 5 ). This is due to the efficient cache use of the Woodbury algorithm as described below. Each BCS needs to process the entire $3 n \times 3 n$ matrix before the next BCS can start. This causes a cache miss for each of the $9 n^{2}$ numbers at every BCS operation that happens $6 m$ times in total. Each miss entails a RAM access which is much slower than a cache access. On the other hand, the full-rank multiplication in the Woodbury formula allows for an implementation letting its $6 \mathrm{~m}$ operations be processed in an inner loop. So, as long as the matrix $V$ fits in cache memory, much fewer cache misses occur resulting in significant speed gain as shown in Fig.5. Here, Matlab's inv function running time for rank-3n matrix inversion which has $\mathcal{O}\left(n^{3}\right)$ operations is also presented for comparison.

The tissue mesh that is used in the simulation consists of 570 nodes and 2801 tetrahedra. The shortest edge is $3.06 \mathrm{~mm}$ and the longest one is $24.04 \mathrm{~mm}$. The minimum and the maximum dihedral angles are $9.94^{\circ}$ and $159.49^{\circ}$, respectively.
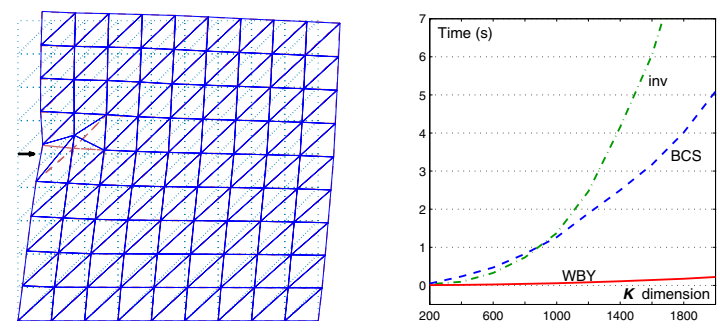

Fig. 5. 2D tissue with a $\boldsymbol{K}$ of dimension 200 (left) and the comparison of mesh modification techniques while increasing the dimension of $\boldsymbol{K}$ (right) 

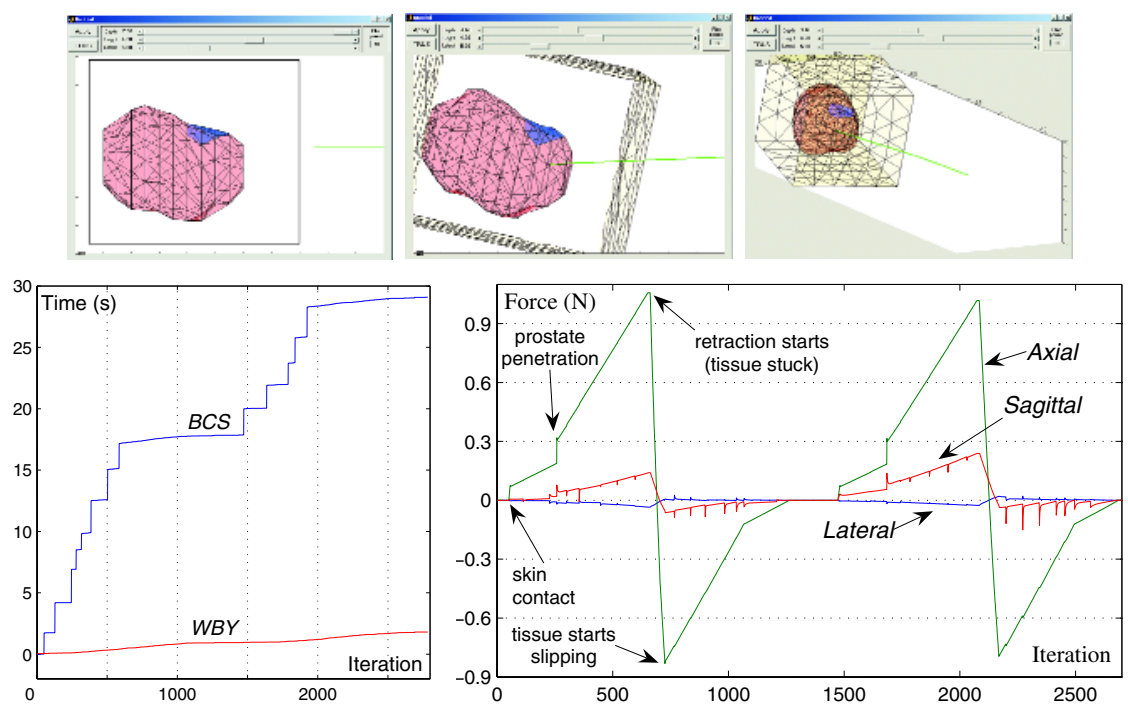

Fig. 6. Interface to the prostate brachytherapy needle insertion simulation (top), cumulative running time since simulation start for two separate instances of needle insertion (lower left), and force on the needle base for Woodbury algorithm (lower right)

Furthermore, only a couple elements have these border-line values showing that the geometry of elements are acceptable for a reasonable FEM approximation.

The 3D needle insertion simulation was implemented in $\mathrm{C}$ for the ease of future use with $\mathrm{VxWorks}{ }^{\circledR}$ which is a real-time operating system used in controlling haptic devices. A Matlab interface, where the needle can be manipulated and the tissue deformation can be observed, was built for testing and debugging purposes (see Fig.6). The three axis components of the needle base force profile during two insertions having different trajectories can be seen in Fig.6

Our first implementation of this simulation used BCS for node repositioning where some iterations took as long as $2.75 \mathrm{~s}$. For an haptic implementation any iteration has to complete in less than $2 \mathrm{~ms}$. That is why the simulation is reimplemented using the Woodbury formula which led us to less than $1 \mathrm{~ms}$ iterations. For the two consecutive insertions that the force profile is given above, the cumulative time elapsed using each method is plotted in Fig.6. The big steps of BCS occur when node repositioning is performed. Since no repositioning is needed during retraction, the cumulative time increases only slightly due to other simulation computations between iterations 650 and 1300.

\section{Conclusions and Future Work}

A prostate brachytherapy simulator design has been presented using a prostate mesh with conforming anatomical boundaries on surfaces generated from contours on parallel slices. The proposed system successfully couples a conform- 
ing quasi-static linear FEM of the tissue with a flexible needle. It is the first physically-based 3D interaction model of a flexible needle insertion into a soft deformable body. The first physically-based 3D simulator of a brachytherapy procedure is programmed with a Matlab interface. The real-time portion of the code is ready to be implemented on a haptic setup. The proposed simulator can also be used for the prediction of prostate motion in brachytherapy planning.

Next, other anatomical structures will be segmented and implemented in the simulator. Particularly, the pubic arch is needed as a fundamental displacement boundary condition. The bladder is also a significant adjacent anatomy to be modeled. Given more anatomical information incorporated into the model, validation of the system will become the next step. TRUS data of the entire procedures were already recorded for this purpose. The prostate shift caused by the needle insertion can easily be seen on those. This observed deformation will be compared with our model for validation when we incorporate at least the pubic arch into our model. Ultimately, the simulation is going to be implemented on a suitable haptic interface. Considering brachytherapy planning, real-time acquisition of the tissue elasticity parameters using elastography which is another future research subject would make accurate intra-op plan adjustments possible when combined with the simulator presented in this paper.

\section{References}

1. Taschereau, R., Pouliot, J., Roy, J., Tremblay, D.: Seed misplacement and stabilizing needles in transperineal permanent prostate implants. Radiotherapy and Oncology 55 (2000) 59-63

2. DiMaio, S.P., Salcudean, S.E.: Needle insertion modeling and simulation. IEEE Transactions on Robotics and Automation 19 (2003) 864-875

3. Gosline, A.H., Salcudean, S.E., Yan, J.: Haptic simulation of linear elastic media with fluid pockets. In: Proceedings of the $12^{\text {th }}$ Symposium on Haptic Interfaces for Virtual Environment and Teleoperator Systems (HAPTICS). (2004) 266-271

4. Nienhuys, H.: Cutting in deformable objects. PhD thesis, Utrecht Univ. (2003)

5. Alterovitz, R., Goldberg, K., Pouliot, J., Taschereau, R., Hsu, I.C.: Sensorless planning for medical needle insertion procedures. In: IEEE/RSJ International Conference on Intelligent Robots and Systems, Las Vegas, NV (2003)

6. Wang, X., Fenster, A.: A haptic-enhanced 3D real-time interactive needle insertion simulation for prostate brachytherapy. In: Proceedings of SPIE Medical Imaging 2004: Vis., Image-Guided Procedures, and Display. Volume 5367. (2004) 781-789

7. Kimura, A.N., Camp, J., Robb, R., Davis, B.: A prostate brachytherapy training rehearsal system - simulation of deformable needle insertion. In: Proceedings of MICCAI. (2002) 264-271

8. Geiger, B.: Three-dimensional modeling of human organs and its application to diagnosis and surgical planning. PhD thesis, École Nationale Supérieure des Mines de Paris, France (1993)

9. Löhner, R.: Progress in grid generation via the advancing front technique. Engineering with Computers 12 (1996) 186-210 